\title{
Prevalência de ansiedade entre estudantes de Farmácia em períodos de avaliações de rendimento acadêmico em Universidade privada na cidade de São Paulo, Brasil
}

\author{
Anxiety prevalence among Pharmacy students during academic performance assessments in a \\ private University in São Paulo, Brazil
}

Prevalencia de ansiedad entre los estudiantes de Farmacia durante los períodos de evaluación del desempeño académico en una Universidad privada en São Paulo, Brasil

\begin{abstract}
Resumo
Ansiedade é considerada crescente na sociedade moderna e relacionada à vida acadêmica, com destaque aos estudantes submetidos às avaliações de rendimento para comprovação de bom desempenho acadêmico. $\mathrm{O}$ objetivo deste estudo foi determinar a prevalência de ansiedade no período de avaliações de rendimento acadêmico, entre estudantes do curso de Farmácia de uma Universidade privada da cidade de São Paulo, Brasil. O estudo foi realizado com 276 graduandos em Farmácia, com idade média entre 18 e 27 anos, (67\%), solteiros (59,8\%) e maioria do gênero feminino (74,6\%). Os estudantes, antes das avaliações de rendimento, referiram ansiedade isolada $(64,5 \%)$ ou associada com estresse, preocupação, insônia e impaciência (63\%). Houve diferença estatística significante entre os estudantes ansiosos $(16,9 \pm 12,2)$ e tranquilos $(3,6 \pm 2,9)$ antes das avaliações de rendimento, com p $<0,001$. Após a realização das avaliações de rendimento, $50 \%$ dos estudantes relatou preocupação isoladamente e a associação de preocupação com impaciência, estresse e insônia foi relatada por 10,1\%. Quanto à frequência ao estudar, 49,3\% relataram que estudam quando há tempo e 45,3\% relataram estudar com frequência. Este trabalho mostra que o ingressar na vida acadêmica é marcado por mudanças acerca de responsabilidades e a escolha de uma profissão, acompanhados de ansiedade e preocupações relacionadas ao bom desempenho, sugerindo a necessidade da adoção de políticas institucionais para prevenção de ansiedade.
\end{abstract}

Palavras-chave: Ansiedade; Estudantes de farmácia; Desempenho acadêmico.

\begin{abstract}
Anxiety is considered increasing in modern society and related to academic life, especially students submitted to performance evaluations to prove good performance. The objective of this study was to determine the prevalence of anxiety in the period of academic performance evaluations, among students of the Pharmacy course of a private University in the city of São Paulo, Brazil. The study was conducted with 276 Pharmacy undergraduates, with an average age between 18 and 27 years (67\%), single (59.8\%) and female prevalence (74.6\%). Students, before performance assessments, reported anxiety alone (64.5\%) or associated with stress, worry, insomnia and impatience $(63 \%)$. There was a statistically significant difference between anxious $(16.9 \pm 12.2)$ and quiet $(3.6 \pm 2.9)$ students before performance evaluations, with $\mathrm{p}<0.001$. After the performance evaluations, $50 \%$ of the students reported concern alone and the association of concern with impatience, stress and insomnia was reported by $10.1 \%$. Regarding the habit of studying, $49.3 \%$ reported that they studied when there was time and $45.3 \%$ reported studying frequently. This paper shows that entering academic life is marked by changes in responsibilities and the choice of a profession, accompanied by anxiety and concerns related to good performance, suggesting the need for the adoption of institutional policies for anxiety prevention.
\end{abstract}

Keywords: Anxiety; Pharmacy students; Academic performance.

\section{Resumen}

La ansiedad se considera creciente en la sociedad moderna y relacionada con la vida académica, especialmente los estudiantes sometidos a evaluaciones de desempeño para demostrar un buen desempeño. El objetivo de este estudio fue determinar la prevalencia de la ansiedad en el período de las evaluaciones de rendimiento académico, entre los estudiantes del curso de Farmacia de una Universidad privada en la ciudad de São Paulo, Brasil. El estudio se realizó con 276 estudiantes universitarios de farmacia, con una edad promedio entre 18 y 27 años (67\%), soltero (59.8\%) y prevalencia femenina $(74.6 \%)$. Los estudiantes, antes de las evaluaciones de desempeño, informaron ansiedad sola 
(64.5\%) o asociada con estrés, preocupación, insomnio e impaciencia (63\%). Hubo una diferencia estadísticamente significativa entre los estudiantes ansiosos $(16,9 \pm 12,2)$ y los tranquilos $(3,6 \pm 2,9)$ antes de las evaluaciones de rendimiento, con $\mathrm{p}<0,001$. Después de las evaluaciones de rendimiento, el $50 \%$ de los estudiantes declararon estar solamente preocupados y la asociación de la preocupación con la impaciencia, el estrés y el insomnio fue declarada por el 10,1\%. Con respecto al hábito de estudiar, el $49.3 \%$ informó que estudiaba cuando había tiempo y el $45.3 \%$ informó que estudiaba con frecuencia. Este documento muestra que ingresar a la vida académica está marcado por cambios en las responsabilidades y la elección de una profesión, acompañado de ansiedad y preocupaciones relacionadas con el buen desempeño, lo que sugiere la necesidad de adoptar políticas institucionales de prevención de la ansiedad.

Palabras clave: Ansiedad; Estudiantes de farmacia; Rendimiento académico.

\section{Introdução}

A ansiedade exibe aumento de manifestações sintomáticas dentre a população (Bandelow \& Michaelis, 2015; WHO, 2017), com destaque aos indivíduos inseridos na vida acadêmica, quer sejam eles estudantes, professores ou técnicos administrativos (Fernandes et al., 2019). Desde a infância, a ansiedade pode ocorrer tanto em alunos com alto ou baixo desempenho acadêmico, com projeções de ansiedade dos pais (Zikopoulou, Kakaniari, Simos, 2019). Alunos que apresentam boas avaliações de rendimento acadêmico podem demonstrar ansiedade devido às expectativas não realistas dos pais, colegas ou mesmo suas, de que devem obter uma excelente performance em todas as disciplinas. Já nos alunos com baixo desempenho nas avaliações de rendimento acadêmico, a ansiedade tende a aumentar caso as situações de fracasso se repitam no ambiente acadêmico constantemente (Wigfield \& Eccles, 1989; Binelli et al., 2012; Nogueira, 2017). A ansiedade, nos momentos de realização da avaliação de rendimento acadêmico, acaba interferindo na capacidade de o aluno recordar ou recuperar um conteúdo aprendido anteriormente, devido ao momento de tensão (Galindo, Moreno, Muñoz, 2009; Figueira, 2013).

A ansiedade também está relacionada com hábitos de estudo e estratégias de aprendizagem, pois os estudantes altamente ansiosos demonstram baixo desempenho escolar. Esse fato pode ser justificado por dois fatores: deficiências nos hábitos de estudo e nas estratégias de aprendizagem utilizadas pelos professores (Costa \& Boruchovitch, 2004; Cardozo et al., 2020).

Estudos realizados com estudantes de medicina demonstraram que a ansiedade costuma aumentar no período da graduação, visto que os universitários demonstram uma dificuldade na adaptação aos novos métodos do processo ensinoaprendizagem. Os estudantes apresentam desgaste emocional maior no período de avaliações de rendimento acadêmico, ocasionado pela quantidade excessiva de conteúdos em diversas disciplinas (Baldassin, Martins, Andrade, 2006; Cunha et al., 2009; Junaid et al., 2020).

Considerando a necessidade de conhecer a prevalência de ansiedade dentre estudantes de um curso de graduação, em períodos anteriores e posteriores à avaliação de rendimento, este estudo foi conduzido com a inclusão de estudantes de Farmácia do primeiro até o oitavo período letivo.

\section{Metodologia}

\subsection{Características gerais}

O presente estudo foi realizado em uma Universidade privada da cidade de São Paulo, em Campus localizado na região oeste da cidade. O objetivo do estudo, a participação voluntária e a garantia de anonimato foram informados pelos pesquisadores e formalizado por meio de um termo de consentimento livre e esclarecido (TCLE), seguindo os preceitos éticos pautados na resolução 196/96 do Conselho Nacional de Saúde (CNS, 1996). O projeto foi aprovado pelo Comitê de Ética da Universidade Nove de Julho sob o parecer de número 319378/2010. 


\subsection{Desenho do estudo}

Trata-se de estudo com delineamento transversal, descritivo, quantitativo e qualitativo (Pereira et al., 2018, p.67). A amostra foi composta por 276 estudantes de ambos os sexos, com faixa etária entre os 18 e 50 anos de idade, considerando os turnos: diurno e noturno, desde o primeiro até o oitavo período do curso de graduação em Farmácia. As informações foram coletadas por meio de um questionário e foram abordados dados como: gênero, idade, costume de estudar para provas, sentimento durante a semana de avaliações e sentimento após as provas.

\subsection{Análise dos dados}

Os dados foram analisados quanto à porcentagem em excel (pacote 365, personal). A análise estatística foi realizada com o auxílio do programa GraphPad InStat na versão 3.1, para comparação entre estudantes ansiosos e tranquilos antes das avaliações de rendimento; e estudantes preocupados e tranquilos após as avaliações de rendimento. Foram consideradas diferenças estatisticamente significantes $(\mathrm{p}<0,05)$, com análise por teste $t$.

\section{Resultados e Discussão}

Neste estudo participaram 276 alunos do $1^{\circ}$ ao $8^{\circ}$ período do curso de Farmácia, com maior número de estudantes do gênero feminino, representando 74,6\% ( $n=206)$ e 25,4\% ( $n=70)$ do gênero masculino. Os estudantes tinham idade média entre 18 e 27 anos (67\%), com predominância de solteiros (59,4\%), conforme demonstra a Tabela 1. 
Tabela 1. Distribuição dos estudantes, segundo as características sociodemográficas (gênero, idade e estado civil), do curso de Farmácia de uma Universidade privada na cidade de São Paulo, Brasil.

\begin{tabular}{lcc}
\hline Características & \multicolumn{2}{c}{ Estudantes } \\
& $\mathrm{n}$ & $\%$ \\
\hline $\begin{array}{l}\text { GÊNERO } \\
\text { Masculino }\end{array}$ & 70 & 25,4 \\
Feminino & 206 & 74,6 \\
Total & 276 & 100
\end{tabular}

\section{Idade (anos)}

$18-22$

$23-27$

$28-32$

$33-37$

$38-42$

$43-50$

Total

\section{ESTADO CIVIL}

\begin{tabular}{lcc} 
casado & 86 & 31,2 \\
solteiro & 164 & 59,4 \\
viúvo & 0 & 0 \\
divorciado & 7 & 2,5 \\
namorando & 19 & 6,9 \\
Total & 276 & 100 \\
\hline
\end{tabular}

Fonte: Autora.
34,8

32,2

19,9

9,4

2,9

0,7

100

276100

A Tabela 1 mostra os dados sociodemográficos do estudo, detalhando gênero, idade e estado civil dos estudantes do curso de Farmácia que participaram da pesquisa.

No estudo foi demonstrado que grande parte dos estudantes, principalmente cursando o $1^{\circ}(80,8 \%)$ e o $2^{\circ}(61 \%)$ períodos, ficam ansiosos momentos antes das avaliações de rendimento acadêmico, conforme explicitado na Tabela 2 . A partir 
de comparação estabelecida entre estudantes ansiosos $(16,9 \pm 12,2)$ e tranquilos $(3,6 \pm 2,9)$ houve diferença estatística significante, com valor de $\mathrm{p}=0,0006$.

Tabela 2. Demonstra as características emocionais dos estudantes do $1^{\circ}$ ao $8^{\circ}$ período do curso de Farmácia no momento anterior às avaliações de rendimento acadêmico.

Comportamento dos estudantes momentos anteriores ao período de avaliações de rendimento acadêmico

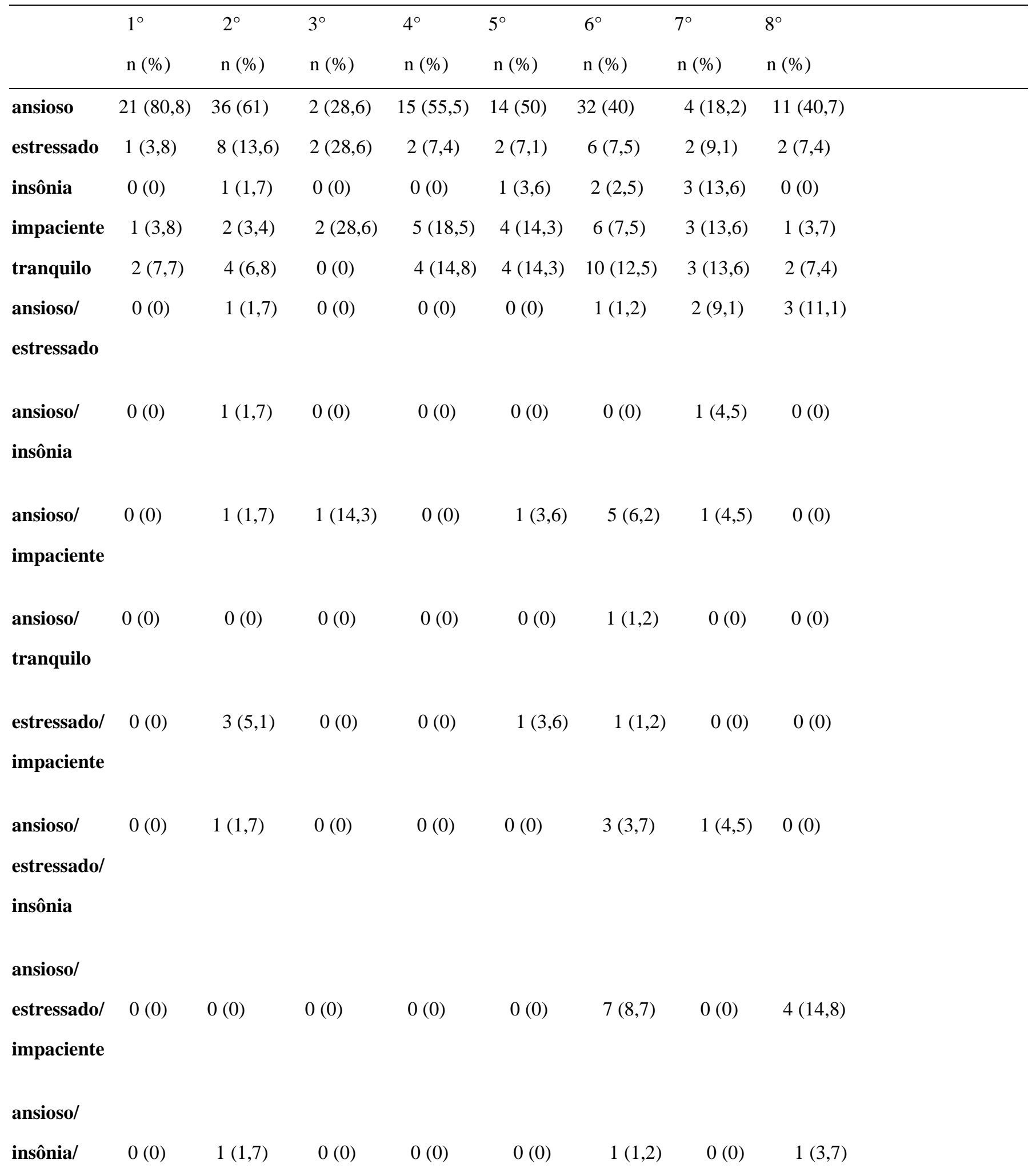


impaciente

estressado/

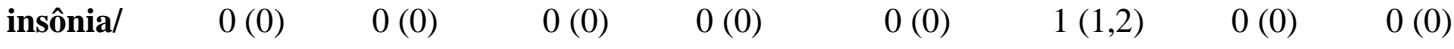

impaciente

ansioso/

stress/

insônia/ $\quad 1(3,8) \quad 0(0) \quad 0(0) \quad 1(3,7) \quad 1(3,6) \quad 4(5,0) \quad 2(9,1) \quad 3(11,1)$

impaciente

Fonte: Elaborado pela autora.

Na Tabela 2 estão expressos os relatos de comportamento dos estudantes do curso de Farmácia antes das avaliações de rendimento acadêmico, a partir de respostas coletadas na pesquisa.

Uma parcela dos estudantes afirmou estado de preocupação em relação ao resultado das avaliações, com destaque aos estudantes do $1^{\circ}, 7^{\circ}$ e $4^{\circ}$ períodos, exibindo os percentuais $73 \%, 59,1 \%$ e $55,5 \%$ respectivamente, conforme explicita a Tabela 3.

Tabela 3. Demonstra as características emocionais dos estudantes do $1^{\circ}$ ao último ano ( $8^{\circ}$ período) do curso de Farmácia no momento posterior às avaliações de rendimento acadêmico.

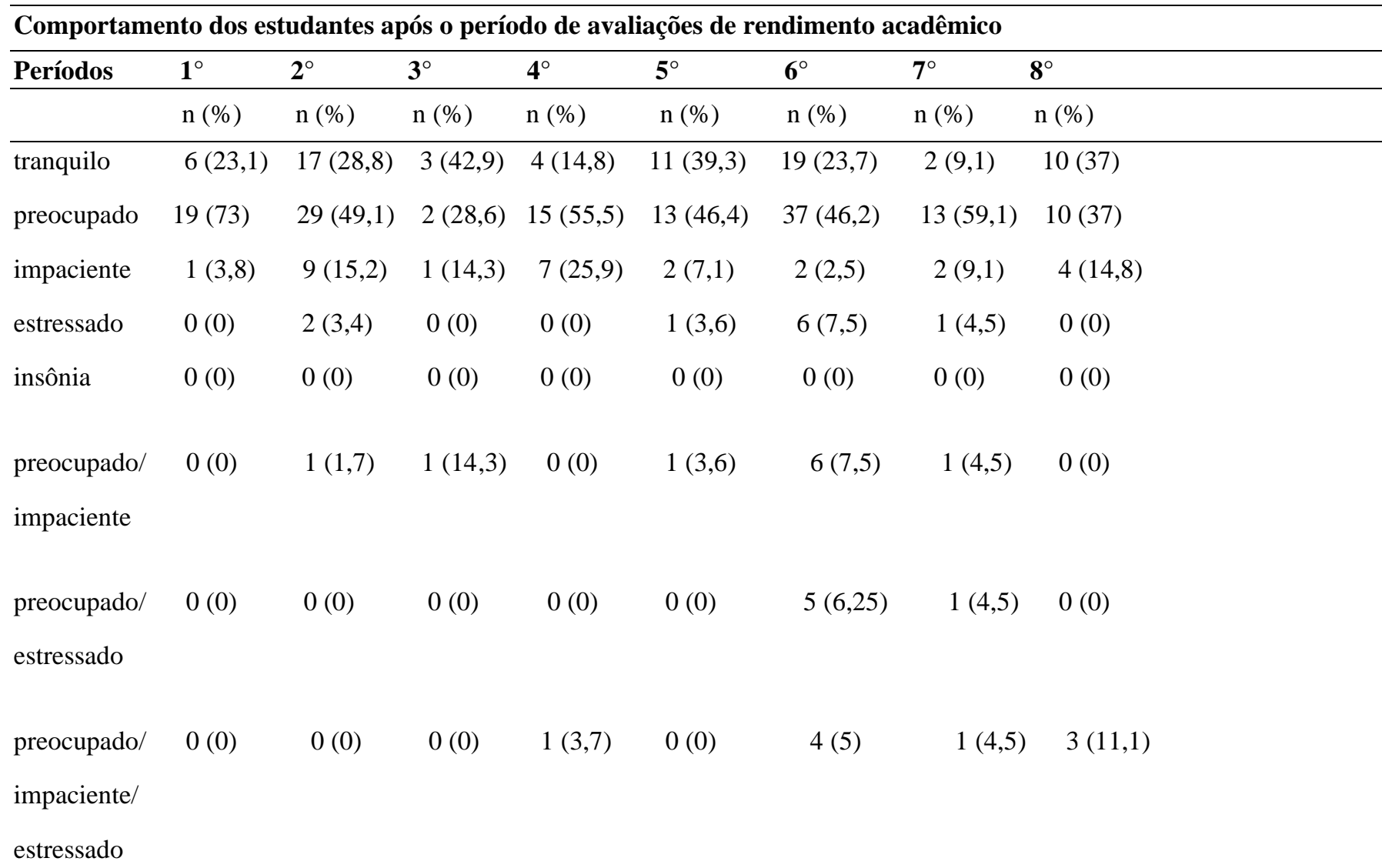




\begin{tabular}{|c|c|c|c|c|c|c|c|c|}
\hline preocupado/ & $0(0)$ & $0(0)$ & $0(0)$ & $0(0)$ & $0(0)$ & $0(0)$ & $1(4,5)$ & $0(0)$ \\
\hline \multicolumn{9}{|l|}{ estressado/ } \\
\hline \multicolumn{9}{|l|}{ insônia } \\
\hline preocupado/ & $0(0)$ & $1(1,7)$ & $0(0)$ & $0(0)$ & $0(0)$ & $1(1,2)$ & $0(0)$ & $0(0)$ \\
\hline \multicolumn{9}{|l|}{ impaciente/ } \\
\hline estressado/ & & & & & & & & \\
\hline nsônia & & & & & & & & \\
\hline
\end{tabular}

Fonte: Autora.

A Tabela 3 aponta os relatos de comportamento dos estudantes do curso de Farmácia em período posterior às avaliações de rendimento realizadas.

Os dados gerais confirmam que os estudantes, antes das avaliações de rendimento, referiram ansiedade isolada (64,5\%) ou associada com estresse, preocupação, insônia e impaciência (63\%). Após a realização das avaliações de rendimento, $50 \%$ dos estudantes relatou preocupação isoladamente e a associação de preocupação com impaciência, estresse e insônia foi relatada por 10,1\%. Já a manifestação de tranquilidade após o término das avaliações de rendimento foi representada por menor percentual; com destaque aos maiores percentuais apresentados por estudantes do $3^{\circ}, 5^{\circ}$ e $8^{\circ}$ períodos, representados por 42,9\%, 39,3\% e 37\% respectivamente. Em comparação estabelecida entre os estudantes que manifestaram preocupação isoladamente $(17,2 \pm 11,06)$ e os estudantes tranquilos $(9,0 \pm 6,4)$ não houve diferença estatística significante $(\mathrm{p}=0,08)$.

Considerando os estudantes do $1^{\circ}$ ao $8^{\circ}$ períodos, 49,3\% afirmam estudar para as provas da universidade quando há tempo, com resultado expressivo entre os estudantes do $2^{\circ}$ e $4^{\circ}$ períodos, exibindo $54,2 \%$ e $63 \%$ respectivamente. Por outro lado, os estudantes do $8^{\circ}$ período afirmaram estudar normalmente para as provas, representando, 59,3\%.

Uma reduzida parcela dos estudantes, representada pelo $2^{\circ}$ e $6^{\circ}$ períodos, com percentual de $6,8 \%$ e $5 \%$ respectivamente, afirmaram não estudar para as avaliações da universidade, pois trabalham e não possuem tempo para estudar. Apenas $1,1 \%$ dos estudantes do $1^{\circ}$ ao $8^{\circ}$ períodos, afirmaram não estudar para as provas, pois não têm ânimo para estudar. 
Tabela 4. Distribuição dos estudantes, segundo o hábito de estudar, do curso de Farmácia de uma Universidade privada na cidade de São Paulo, Brasil.

\section{Perfil sobre a frequência de estudo às avaliações de rendimento acadêmico}

\begin{tabular}{|c|c|c|c|c|}
\hline & \multicolumn{2}{|c|}{ sim, quando há tempo } & \multicolumn{2}{|c|}{ sim, estudo normalmente às avaliações } \\
\hline & $\mathrm{n}$ & $\%$ & $\mathrm{n}$ & $\%$ \\
\hline $1^{\circ}$ per. & 13 & 50 & 13 & 50 \\
\hline $2^{\circ}$ per. & 32 & 54 & 23 & 39 \\
\hline $3^{\circ}$ per. & 5 & 71,4 & 1 & 14,3 \\
\hline $4^{\circ}$ per. & 17 & 63 & 9 & 33,3 \\
\hline $5^{\circ}$ per. & 13 & 46,4 & 13 & 46,4 \\
\hline $6^{\circ}$ per. & 35 & 43,7 & 40 & 50 \\
\hline $7^{\circ}$ per. & 11 & 50 & 10 & 45,4 \\
\hline $8^{\circ}$ per. & 10 & 37 & 16 & 59,3 \\
\hline \multirow[t]{3}{*}{ Total } & 136 & 49,3 & 125 & 45,3 \\
\hline & \multicolumn{2}{|c|}{ não, pois trabalho e não sobra tempo para estudar } & \multicolumn{2}{|c|}{ não, pois não tenho ânimo para estudar } \\
\hline & $\mathrm{n}$ & $\%$ & $\mathrm{n}$ & $\%$ \\
\hline $1^{\circ}$ per. & 0 & 0 & 0 & 0 \\
\hline $2^{\circ}$ per. & 4 & 6,8 & 0 & 0 \\
\hline $3^{\circ}$ per. & 1 & 14,3 & 0 & 0 \\
\hline $4^{\circ}$ per. & 0 & 0 & 1 & 3,7 \\
\hline $5^{\circ}$ per. & 2 & 7,1 & 0 & 0 \\
\hline $6^{\circ}$ per. & 4 & 5 & 1 & 1,2 \\
\hline $7^{\circ}$ per. & 0 & 0 & 1 & 4,5 \\
\hline $8^{\circ}$ per. & 1 & 3,7 & 0 & 0 \\
\hline Total & 12 & 4,3 & 3 & 1,1 \\
\hline
\end{tabular}

Fonte: Autora.

A Tabela 4 explicita o perfil de frequência ao ato de estudar, a partir de respostas dos estudantes do curso de Farmácia que participaram da pesquisa, considerando as opções: (1) sim, quando há tempo; (2) sim, estudo normalmente às avaliações; (3) não, pois trabalho e não sobra tempo para estudar; (4) não, pois não tenho ânimo para estudar.

Os resultados apontam dados com relação à manifestação de ansiedade em momento anterior e posterior às avaliações. Nota-se que os estudantes do primeiro ano de graduação apresentaram maior ansiedade relatada antes das avaliações de rendimento, que se projetam à realização da avaliação e os impactos no processo de resolução de uma avaliação de rendimento. De modo complementar, os estudantes do primeiro período manifestaram maior preocupação no processo de espera da nota relativa à avaliação de rendimento concluída; sinalizando que esta fase marca o processo de transição entre o ensino médio e a vida acadêmica. Neste sentido, há diferenças entre estratégias e métodos de ensino-aprendizagem, em que 
esta etapa da formação se caracteriza por um processo de adaptação ao novo sistema. Ao mesmo tempo, estudantes do sétimo período manifestaram, majoritariamente, preocupação no processo de espera do resultado da avaliação de rendimento concluída. Este aspecto sinaliza um comportamento no último ano da graduação, decisivo ao término do curso. Diante da associação de manifestações entre sentimentos e percepções, o percentual expresso foi menor em todas as situações apontadas nas Tabelas 2 e 3 .

Com relação à pergunta sobre a frequência de estudo para as avaliações de rendimento, 49,3\% do total de estudantes afirmou que estuda quando possui tempo e 45,3\% afirmou que estuda normalmente às avaliações. Dentre os que estudam com frequência às avaliações, os estudantes do último período manifestaram maior percentual, representado por 59,3\%.

Estes dados reforçam diferenças existentes entre os anos da graduação, com destaque às mudanças no processo de ingresso à vida acadêmica, assim como empenho de estudantes concluintes para finalizar a graduação a partir do cumprimento dos requisitos associados às avaliações de rendimento para obtenção do grau de bacharel em Farmácia. Neste sentido, programas de prevenção às alterações provenientes do comportamento e da emocionalidade compõem uma estratégia para a redução de ansiedade e estresse. Rith-Najarian et al. (2019) colocam o benefício de programas preventivos produzindo uma redução moderada de sintomas de ansiedade e estresse. De modo complementar, Huang et al. (2019) confirmaram que intervenções como: terapia cognitiva comportamental, baseadas na atenção, arteterapia, exercício e apoio de colegas; foram eficazes em efeito medido pós-intervenção para estudantes com transtorno de ansiedade generalizada.

\section{Considerações Finais}

Os dados encontrados na presente pesquisa demonstraram que os estudantes do curso de Farmácia apresentaram ansiedade durante o período da graduação, associado com o estresse, preocupação, insônia e impaciência. É visível que o sistema tradicional de avaliação de rendimento promove alterações comportamentais que denotam ansiedade. Neste sentido, vale uma revisão e reavaliação das estratégias e abordagens metodológicas adotadas pelos professores. De modo complementar, a adoção de medidas preventivas e de intervenção permanente no contexto universitário representam estratégias que colaboram à redução de ansiedade manifestada por estudantes universitários.

Para o estudante, o desempenho insatisfatório resulta em redução da autoestima e desânimo. Isso reflete no aumento da ansiedade, da impaciência e da preocupação, visto que, a responsabilidade é grande dos universitários, pois todos almejam concluir a graduação. Considerando o sistema tradicional de ensino, mudanças nas relações do processo ensino-aprendizagem são requeridas, garantindo a humanização como eixo central, aliada à elaboração e à implantação de políticas institucionais de prevenção, promoção e recuperação da saúde mental voltadas aos estudantes de graduação.

\section{Agradecimentos}

Agradeço ao egresso Thiago Gonçalves de Oliveira pelo auxílio na coleta dos dados.

\section{Referências}

Baldassin, S., Martins, L. C., \& Andrade, A. G. (2006). Traços de ansiedade entre estudantes de medicina. Arquivos Médicos do ABC, 31(1), 27-3.

Bandelow, B., \& Michaelis, S. (2015). Epidemiology of anxiety disorders in the 21st century. Dialogues in Clinical Neuroscience, 17(3), 327-35.

Binelli, C., Ortiz, A., Muñiz, A., Gelabert, E., Ferraz, L., Filho, A., Crippa, J.A.S., Nardi, A. E., Subirà, S., \& Martin-Santos, R. (2012). Social anxiety and negative early life events in university students. Revista Brasileira Psiquiatria, 34(Supl.1), S69- 74.

Cardozo, L. T., Azevedo, M. A. R., Carvalho, M. S. M., Costa, R., Lima, P. O., \& Marcondes, F. K. (2020). Effect of an active learning methodology combined with formative assessments on performance, test anxiety, and stress of university students. Advances in Physiology Education, 44(4), 744-751.

Costa, E. R., \& Boruchovitch, E. (2004). Compreendendo relações entre estratégias de aprendizagem e a ansiedade de alunos do ensino fundamental de Campinas. Psicologia: Reflexão e Crítica, 17(1), 15-24. 
Research, Society and Development, v. 10, n. 2, e25610212526, 2021

(CC BY 4.0) | ISSN 2525-3409 | DOI: http://dx.doi.org/10.33448/rsd-v10i2.12526

Cunha, M. A. B., Neves, A. A. F., Moreira, M. E., Hehn, F. J., Lopes, T. P., Ribeiro, C. C. F., \& Watanabe, A. P. F. (2009). Transtornos psiquiátricos menores e procura por cuidados em estudantes de medicina. Revista Brasileira de Educação Médica, 33 (3), 321-328.

Fernandes, I. M. C., Ribeiro, A. M., Gomes, R. L., Lopes, J. S. S., Vanderlei, L. C. M., \& Lorençoni, R. M. R. (2019). Anxiety, depression and stress among employees of a public higher education institution in São Paulo, Brazil. Revista Brasileira de Medicina do Trabalho, 17(4), 530-536.

Figueira, C. (2013). Bem-estar nos estudantes do ensino superior: papel das exigências e dos recursos percebidos no contexto académio e das actividades de voluntariado. Universidade de Lisboa.

Galindo, S., Moreno, I., \& Muñoz, J. (2009). Prevalencia de ansiedad y depresión en una población de estudiantes universitarios: factores académicos y sociofamiliares asociados. Clínica y Salud, 20(2), 177-187.

Huang, J., Nigatu, Y. T., Smail-Crevier, R., Zhang, X., \& Wang, J. (2018). Interventions for common mental health problems among university and college students: A systematic review and meta-analysis of randomized controlled trials. Journal Psychiatric Research, 107, 1-10.

Junaid, M. A. L., Auf, A. I., Shaikh, K., Khan, N., \& Abdelrahim, S. A. (2020). Correlation between Academic Performance and Anxiety in Medical Students of Majmaah University - KSA. Journal of Pakistan Medical Association, 70(5), 865-868.

Nogueira, M. J. C. (2017). Saúde mental em estudantes do ensino superior: fatores protetores e fatores de vulnerabilidade. Tese (Doutorado). Universidade de Lisboa. 269 p.

Pereira, A. S., Shitsuka, D. M., Parreira, F. J., Shitsuka, R. (2018). Metodologia da pesquisa científica. UFSM. https://repositorio.ufsm.br/bitstream/handle/1/15824/Lic_Computacao_Metodologia-Pesquisa-Cientifica.pdf?sequence=1.

Rith-Najarian, L. R., Boustani, M. M., \& Chorpita, B. F. (2019). A systematic review of prevention programs targeting depression, anxiety, and stress in university students. Journal of Affective Disorders, 257, 568-584.

World Health Organization (WHO). (2017). Depression and Other Common Mental Disorders: Global Health Estimates. WHO: Geneve, 24 p.

Zikopoulou, O.; Kakaniari, K.;\& Simos, G. (2019). Assessing anxiety symptoms in children: Agreement between parents and children. Psychiatr 